\title{
Self-avoiding random walks: Some exactly soluble cases
}

\author{
Deepak Dhar \\ George W. Downs Laboratory of Physics, California Institute of Technology, Pasadena, California 91125 \\ (Received 12 July 1977) \\ We use the exact renormalization group equations to determine the asymptotic behavior of long self- \\ avoiding random walks on some pseudolattices. The lattices considered are the truncated 3 -simplex, the \\ truncated 4-simplex, and the modified rectangular lattices. The total number of random walks $C_{n}$, the \\ number of polygons $P_{n}$ of perimeter $n$, and the mean square end to end distance $\left\langle R_{n}^{2}\right\rangle$ are assumed to \\ be asymptotically proportional to $\mu^{n} n^{\gamma-1}, \mu^{n} n^{a-3}$, and $n^{2 v}$ respectively for large $n$, where $n$ is the total \\ length of the walk. The exact values of the connectivity constant $\mu$, and the critical exponents $\lambda, \alpha, v$ \\ are determined for the three lattices. We give an example of two lattice systems that have the same \\ effective nonintegral dimensionality $3 / 2$ but different values of the critical exponents $\gamma, \alpha$, and $\nu$.
}

\section{INTRODUCTION AND OUTLINE}

The self avoiding random walk was originally proposed as a model of polymer chains, to study the effect of excluded volume. ${ }^{1}$ The properties of such walks are connected with some properties of the Ising model. ${ }^{2}$ The study of the configurational problems encountered in this problem may be expected to shed some light on the more general problem of second order phase transitions. The problem has been attached using a variety of analytical and numerical techniques, ${ }^{3-5}$ but the number of exact results known is small. It has resisted a complete solution in the physically interesting case of three dimensions, or even in the considerably simpler case of two dimensions.

It is thus of some interest to study the problem for some pseudolattices, where the exact solution may be worked out and its behavior analyzed in detail. An example of such lattices is the Bethe lattice, which has been very important historically in the development of the theory of phase transitions. Detailed study of the Ising model on this lattice has suggested the possibility of a new kind of phase transition (phase transitions of continuous order), ${ }^{6}$ which have subsequently been realized on more conventional lattices. ${ }^{7}$

Part of the motivation for the study of pseudolattices springs from the fact that they are very good pedagogical examples of renormalization group techniques at work. Despite enormous progress in the application of renormalization techniques to the field of phase transitions since the pioneering work of Kadanoff and Wilson, ${ }^{8}$ the number of cases which show nontrivial phase transitions and where the exact renormalization transformation may be explicitly implemented, has remained rather small. The only other exceptions are the Gaussian model ${ }^{9}$ and the hierarchial model. ${ }^{10}$ These lattices may also be used to test the validity of new approximation schemes.

The problem of self avoiding random walks on a Bethe lattice is trivial, of course, because of the absence of any closed loops. In this paper we study the self avoiding random walk problem for the truncated tetrahedron lattice, the truncated 4-simplex lattice, and the modified rectangular lattice.

The truncated tetrahedron lattice was defined by Nelson and Fisher. ${ }^{11}$ In a previous paper ${ }^{12}$ (hereafter referred to as I) we have generalized their construction to define the truncated $n$-simplex lattice for arbitrary integer $n$, and shown that the effective dimensionality of this lattice is $2 \ln (n) / \ln (n+2)$. We have also defined the modified rectangular lattice, which is planar and is obtained by deleting some bonds from a planar square lattice. For details of the construction of these lattices, the reader is referred to $I$. In the following discussion familiarity with its contents is assumed.

These lattices are defined recursively, and the exact renormalization equations may be written down for these lattices in terms of only a small number of coupling constants. In I, we used this property to determine the critical behavior of the classical $X Y$ model (the results may easily be extended to arbitrary integral spin dimensionality) and the Fortuin-Kasteleyn cluster model on these lattices. No phase transition at a finite (nonzero) temperature is found. The analysis in this paper differs from I mainly in that for the self avoiding random walk problems, the recursion equations have nontrivial fixed points, even for lattices with effective dimensionality less than two. The system shows a phase transition in the sense that the generating functions of the random walk become singular as a function of their argument. We can determine the critical exponents using the standard renormalization group techniques. The recursion equations are coupled algebraic equations and their derivation and analysis is quite straightforward. In the parameter space of the coupling constants, we observe the phenomena of the point specifying the effective interaction approaching the fixed point of renormalization transformation initially. Eventually the point escapes away from the fixed point after a large number of iterations unless the starting system was exactly critical. Linearizing the recursion equations about the fixed point, we determine the critical exponents from the eigenvalues of the linearized renormalization transformation matrix.

\section{PRELIMINARIES AND NOTATION}

A self avoiding random walk on lattice is a random walk with the constraint that no lattice point is visited more than once. We associate weight factor $x$ with each step of the random walk and define the generating functions

$$
C(x)=\lim _{N \rightarrow \infty} \frac{1}{N} \sum_{n=1}^{\infty} C_{n}(N) x^{n}
$$




$$
P(x)=\lim _{N \rightarrow \infty} \frac{1}{N} \sum_{n=2}^{\infty} P_{n}(N) x^{n} .
$$

Here $C_{n}(N)$ is the total number of distinct self avoiding randon walks of $n$ steps on a large lattice consisting of $N$ lattice points. $P_{n}(N)$ is the number of distinct closed simple polygons of perimeter $n$ on the lattice. The random walks may start from any point on the lattice. For large $N$, the numbers $C_{n}(N)$ and $P_{n}(N)$ are asymptotically proportional to $N$, and the limit exists. For regular lattices, where all the lattice point are equivalent, this limiting procedure is unnecessary because the number of self avoiding walks of length $n$ is independent of the vertex from which the walk starts (so long as the starting vertex is not too close to the boundary of the lattice). This is not the case for the spatially inhomogeneous lattices studied here, and the averaging over all possible positions of the starting point is necessary. We define

$$
\begin{aligned}
& \bar{P}_{n}=\lim _{N \rightarrow \infty} P_{n}(N) / N, \\
& \bar{C}_{n}=\lim _{N \rightarrow \infty} C_{n}(N) / N o
\end{aligned}
$$

We know that for large $n, \bar{P}_{n}$ and $\bar{C}_{n}$ increase geometrically with $n$. Let us assume that for large $n$

$$
\begin{aligned}
& \bar{P}_{n} \simeq K_{1} \mu^{n} n^{\alpha-3}, \\
& \bar{C}_{n} \simeq K_{2} \mu^{n} n^{\gamma-1},
\end{aligned}
$$

where $K_{1}$ and $K_{2}$ are some coefficients of proportionality. In general, we represent a constant of proportionality by $K$, with or without subscripts. Its numerical value is not necessarily the same in different equations. $\mu$ is called the connectivity constant of the lattice and $\alpha$ and $\gamma$ are the critical indices for the random walk. Substituting the asymptotic behavior of $C_{n}$ and $P_{n}$ in Eq. (2) we find that as $x$ tends to $1 / \mu$ from below, the asymptotic behavior of $C(x)$ and $P(x)$ is given by

$$
\begin{aligned}
& C(x) \simeq K_{2}(1-x \mu)^{-\gamma}+\text { less singular terms, } \\
& P(x) \simeq K_{1}(1-x \mu)^{2-\alpha}+\text { less singular terms. }
\end{aligned}
$$

The average number of self avoiding walks per site that return to the origin (polygonal closures) after exactly $n$ steps is given by $2 n \bar{P}_{n}$. We also define the generating function for the mean squared end to end distance by

$$
R(x)=\lim _{N \rightarrow \infty} \frac{1}{N} \sum_{L}[R(L)]^{2} x^{n(L)},
$$

where $R(L)$ is the end to end distance for the random walk $L$ with total number of steps given by $n(L)$. The summation extends over all possible self avoiding random walks $L$ on a large lattice of size $N$. We define the critical exponent $\nu$ by the relation

$$
\left\langle R_{n}^{2}\right\rangle \approx K n^{2 \nu} \text { for large } n,
$$

where $\left\langle R_{n}^{2}\right\rangle$ is the mean squared end to end distance for $n$-step self avoiding random walks, all walks being weighted equally. Since the number of such walks increases as $\mu^{n} n^{r-1}[\mathrm{Eq}$. (6)], we find that the asymptotic behavior of $R(x)$ as $x \mu-1$ from below is given by

$$
R(x) \sim K(1-x \mu)^{-\gamma-2 \nu}+\text { less singular terms. }
$$

In the next section, we use the renormalization group techniques to determine the values of the constants $\mu, \alpha, \gamma$, and $\nu$ for the truncated 3-simplex, the truncated 4-simplex lattice and the modified rectangular lattices, by determining the singular behavior of their generating functions $C(x), P(x)$, and $R(x)$. We show that for the truncated 3-simplex lattice

$$
\mu=1.6180, \quad \alpha=0.7342, \quad \gamma=1.3752, \quad \nu=0.7986 \text {. }
$$

For the modified rectangular lattice

$$
\mu=1.6909, \quad \alpha=0.6699, \quad \gamma=1.4403, \quad \nu=0.6650 .
$$

And for the truncated 4-simplex lattice

$$
\mu=2.2866, \quad \alpha=0.5413, \quad \gamma=1.4461, \quad \nu=0.7294 \text {. }
$$

\section{CALCULATION OF THE CRITICAL EXPONENTS}

In this section we derive the connectivity constants and the critical indices mentioned in the previous section. The analysis of all the three lattices is quite similar and some of the details in the treatment of the truncated 4-simplex lattice and the modified rectangular lattice have been omitted. The treatment may be extended to other recursively defined pseudolattices, but the number of variables that have to be considered to form a closed set of recursion equations soon becomes very large and the analysis becomes difficult.

\section{A. The truncated 3-simplex lattice}

We would like to determine the behavior of the generating functions of the random walks $C(x), P(x)$, and $R(x)$ for the truncated 3-simplex lattice. These functions are weighted sums over self avoiding walks. The weight of a walk of length $n$ is $x^{\eta}$. [For $R(x)$, there is an additional multiplicative weight factor depending on the end to end distance of the walk, Eq. (9)]. Instead of assigning a weight $x$ to each step of the walk, we may equivalently assign a weight $x$ to each vertex that the walk passes through, and a weight $\sqrt{\bar{x}}$ to each of the two vertices that are the starting or the end of the walk. Then, for example, $P(x)$ is the sum over all possible configurations with a single loop.

The renormalization transformation consists of summing over all the internal configurations of the $r$ th order triangles, as was done for the Fortuin-Kasteleyn cluster model in $\mathrm{I}$. We define the rth order restricted partition function as shown in Fig。1. Here $A^{(r)}$ is the weight of an $r$ th order triangle with one line going in. The end point of the line may be any of the vertices

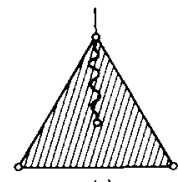

$A^{(r)}$

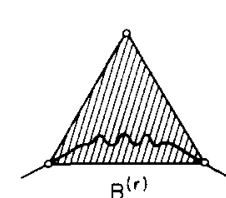

$\mathrm{B}^{(r)}$

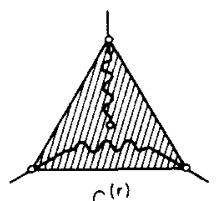

$c^{(r)}$

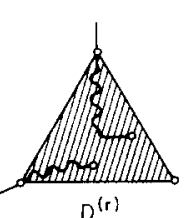

$\mathrm{D}^{(\mathrm{r})}$
FIG. 1. Restricted partition functions for an $r$ th order triangle. The shaded triangles denote $r$ th order triangles, of which only the comer vertices and the end points of the self-avoiding walks are shown. 

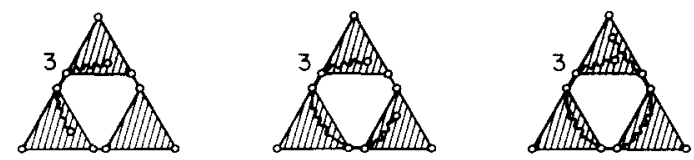

FIG. 2. All possible configurations of an open self avoiding walk of order $r$. The shaded triangles denote $(r-1)$ th order graphs of which only the corner vertices are shown. The factor 3 is for the three possible orientations of the figures.

inside the $r$ th order triangle. We sum over all possible configurations of the $r$ th order triangle consistent with the constraint that one of the end points of the walk lies inside the $r$ th order triangle. Similarly $B^{(r)}$ is the weight of an $r$ th order triangle in which a line goes in from one of the corner vertices and comes out from the other. (The lines are undirected. We use the term going in and coming out rather loosely.) The weights $C^{(r)}$ and $D^{(r)}$ are defined similarly。 The starting values of these weights are

$$
\begin{aligned}
& A^{(0)}=\sqrt{x}, \\
& B^{(0)}=x, \\
& C^{(0)}=D^{(0)}=0 .
\end{aligned}
$$

We call a closed or open walk $L$ of order $r$ if $r$ is the minimum value of $p$ such that $L$ can be completely described inside a $p$ th order triangle. The sum of weights of all $r$ th order closed loops inside one $r$ th order triangle is clearly $\left(B^{(r-1)}\right)^{3}$. Since there are $3^{r}$ points in each $r$ th order triangle, the contribution of $r$ th order closed loops per site is $\left(B^{(r-1)}\right)^{3} / 3^{r}$. Hence we get

$$
P(x)=\sum_{r=1}^{\infty} 3^{-r}\left(B^{(r-1)}\right)^{3} .
$$

Similarly we get (Fig, 2)

$$
\begin{aligned}
C(x)= & \sum_{r=1}^{\infty} 3^{-r}\left[3 A^{(r-1)^{2}}+3 B^{(r-1)}\left(A^{(r-1)}\right)^{2}\right. \\
& \left.+3\left(B^{(r-1)}\right)^{2} D^{(r-1)}\right]
\end{aligned}
$$

It is easy to write down the recursion equations for the weights $A^{(r)}, B^{(r)}, C^{(r)}, D^{(r)}$ by drawing graphically all possible ways a configuration of $(r+1)$ th order triangle may arise out of the configurations of $(r)$ th order triangles. Figure 3 shows all the possible configurations that contribute to $B^{(r+1)}$. This shows that

$$
B^{(r+1)}=\left(B^{(r)}\right)^{2}+\left(B^{(r)}\right)^{3} \text { 。 }
$$

The recursion equations for $A^{(r)}, C^{(r)}$, and $D^{(r)}$ are written down similarly and we get

$$
\begin{aligned}
& A^{(r+1)}=A\left(1+2 B+2 B^{2}\right)+C\left(2 B^{2}\right), \\
& C^{(r+1)}=A\left(B^{2}\right)+C\left(3 B^{2}\right), \\
& D^{(r+1)}=\left(A^{2}+2 A^{2} B+4 A B C+6 B C^{2}\right)+D\left(2 B+3 B^{2}\right),
\end{aligned}
$$

where we have suppressed the superscripts of $A^{(r)}$, $B^{(r)}, C^{(r)}$, and $D^{(r)}$ in the right-hand sides of Eqs. (16b)-(16d).

Equations (13)-(16) determine the functions $P(x)$ and
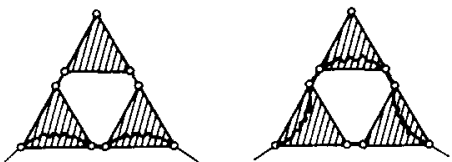

FIG. 3. All possible configurations of $\boldsymbol{r}$ th order triangles (denoted by shaded triangles in the diagram) that contribute to $B^{(r+1)}$

$C(x)$ completely. We also notice that the recursion equation for $B^{(r+1)}$ involves only $B^{(r)}$. Also the recursion equations for $A^{(r+1)}$ and $C^{(r+1)}$ are independent of $D^{(r)}$.

From Eq。(16a) and (14), we see that the $P(x)$ satisfies the functional equation

$$
P(x)=\frac{x^{3}}{3}+\frac{1}{3} P\left(x^{2}+x^{3}\right) .
$$

This equation has fixed points given by the equation

$$
x^{*}=x^{*^{2}}+x^{*^{3}}
$$

which gives the fixed points $x^{*}=0,(\sqrt{5}-1) / 2, \infty$ in the allowed (real nonnegative) range of $x$. The fixed points $x^{*}=0$ and $x^{*}=\infty$ are attractive fixed points while the point $x^{*}=(\sqrt{5}-1) / 2$ is repulsive. If the starting value $B^{(0)}$ is less than $(\sqrt{5}-1) / 2$, from Eq. (16a) we see that with successive iterations, the value of $B^{(r)}$ decreases to zero. If $B^{(0)}$ is greater than $(\sqrt{5}-1) / 2$, for large $r, B^{(r)}$ tends to infinity and $P(x)$ is infinite. This shows that the connective constant of this lattice is given by

$$
\mu=2 /(\sqrt{5}-1) \cong 1.6180 \text {. }
$$

Putting $x=1 / \mu$ in Eq. (17) we get

$$
P\left(\mu^{-1}\right)=\mu^{-3} / 2 \text {. }
$$

Consider $x=\mu^{-1}-\delta$ where $\delta$ is a small positive number. Then Eq. (17) gives

$$
P\left(\mu^{-1}-\delta\right)=\frac{1}{3} \mu^{-3}-\mu^{-2} \delta+\frac{1}{3} P\left(\mu^{-1}-\delta\left(2+\mu^{-2}\right)\right)+O\left(\delta^{2}\right) .
$$

We assume that the singular part of $P\left(\mu^{-1}-\delta\right)$ varies as $\delta^{2-\alpha}$. This gives us, from Eq. (20),

$$
\alpha=2-\ln 3 / \ln \left(2+\mu^{-2}\right) \simeq 0.7342 .
$$

Let us define

$$
\delta^{(r)}=\mu^{-1}-B^{(r)} \text {. }
$$

Then to lowest order in $\delta, \mathbf{E q}$. (16a) gives the recursion relation

$$
\delta^{(r+1)}=\left(2+\mu^{-2}\right) \delta^{(r)} .
$$

We choose a small positive number $\epsilon$, and choose a starting value $\delta^{(0)}$ sufficiently small so that

$$
1 \gg \epsilon \gg \delta^{(0)}
$$

and

$$
r_{0} \stackrel{\text { def }}{=} \ln \left(\epsilon / \delta^{(0)}\right) / \ln \left(2+\mu^{-2}\right) \gg 1 .
$$

Then for $r<r_{0}, \delta^{(r)}$ is less than $\epsilon$ and we may replace $B^{(r)}$ in Eqs. (16b)-(16d) by $\mu^{-1}$. This gives us a set of coupled linear recursion equations for the constants $A^{(r)}$ and $C^{(r)}$, 


$$
\begin{aligned}
& A^{(r+1)} \approx A^{(r)}\left(1+2 \mu^{-1}+2 \mu^{-2}\right)+C^{(r)} 2 \mu^{-2}, \\
& C^{(r+1)} \approx A^{(r)} \mu^{-2}+C^{(r)} 3 \mu^{-2},
\end{aligned}
$$

which imply that

$$
\begin{aligned}
& A^{(r)} \approx K_{1} \lambda^{r}, \\
& C^{(r)} \approx K_{2} \lambda^{r} \text { for } 1<r<r_{0},
\end{aligned}
$$

where $K_{1}$ and $K_{2}$ are some constants of proportionality. $\lambda$ is the larger eigenvalue of the matrix

$$
\left[\begin{array}{rr}
1+2 \mu^{-1}+2 \mu^{-2} & 2 \mu^{-2} \\
\mu^{-2} & 3 \mu^{-2}
\end{array}\right]
$$

which gives

$$
\lambda=\left[3+3 \mu^{-2}+\left(9-18 \mu^{-2}+17 \mu^{-4}\right)^{1 / 2}\right] / 2 .
$$

Substituting from Eqs. (27a)-(27b) into Eq. (16d), we see that the recursion equation for $D^{(r+1)}$ has the form

$$
D^{(r+1)} \approx K \lambda^{2 r}+D^{(r)}\left(2+\mu^{-2}\right) .
$$

Since $\lambda^{2}$ is greater than $\left(2+\mu^{-2}\right)$, we see that this equation implies

$$
D^{(r)} \approx K_{1} \lambda^{2 r} \text { for } 1<r<r_{0} \text {. }
$$

For $r>r_{0}$, the constants $B^{(r)}$ and $C^{(r)}$ rapidly approach zero and the constants $A^{(r)}$ and $D^{(r)}$ tend to finite asymptotic values approximately given by

$$
\begin{aligned}
& A^{(r)} \approx K_{1}(\epsilon) \lambda^{r_{0}} \text { for } r>r_{0}, \\
& D^{(r)} \approx A^{(r)^{2}} \text { for } r>r_{0} .
\end{aligned}
$$

Here $K_{1}(\epsilon)$ is again a constant of proportionality which depends on $\epsilon$, but is independent of $\delta$. We substitute these values from Eqs. (31) and (27) in Eq. (15), and approximating the sum by its largest term we see that

$$
C(x) \sim K \frac{\lambda^{2 r_{0}}}{3^{r_{0}}} .
$$

Substituting for $r_{0}$ from Eq. (25) we get

$$
C(x) \sim K\left(\frac{\epsilon}{\delta}\right)^{\gamma}
$$

with

$$
\gamma=\ln \left(\lambda^{2} / 3\right) / \ln \left(2+\mu^{-2}\right) \approx 1.3752 \text { 。 }
$$

In Eq. (33), the constant of proportionality $K$ must vary as $\epsilon^{-\gamma}$; so that $C(x)$ is independent of $\epsilon$ as should be obvious from its definition.

The critical exponent $\nu$ may be determined similarly. We note that for $r<r_{0}$, the contribution of the $r$ th order open loops to $R(x)$ is approximately

$$
\left(2^{r}\right)^{2} K \lambda^{2 r} / 3^{r} \text {. }
$$

For $r>r_{0}$, the coefficient $B^{(r)}$ rapidly become zero. And in a configuration of the type $A^{(r)}$ (Fig. 1) the endpoint of the line stays close to the corner vertex from which it entered the triangle. Thus for $r>r_{0}$, the contribution of the $r$ th order open loops to $R(x)$ varies as $K\left(4 \lambda^{2}\right)^{r_{0}} 3^{-r}$ so that we have

$$
R(x) \sim K\left\{(4 \lambda)^{2} / 3\right\}^{r_{0}} \text {. }
$$

Substituting for $r_{0}$ from Eq. (25) and comparing its

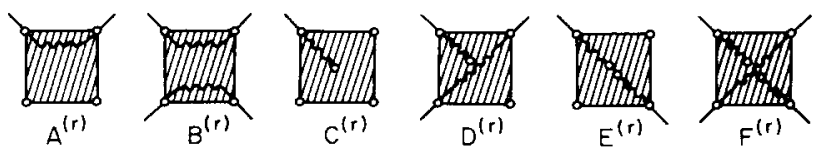

FIG. 4. The restricted partitions for an $r$ th order square. Only the corner vertices of the square and the connections of the walks joining them are shown.

dependence on $\delta$ with Eq. (11) we get

$$
\nu=\frac{\ln 2}{\ln \left(2+\mu^{-2}\right)} \approx 0.7986 \text {. }
$$

This determines all the critical exponents $\alpha, \gamma, \nu$ 。 We remark here that though a more complete and rigorous analysis of the recursion equations is certainly possible, it is unnecessary since all the constants $\mu, \alpha, \gamma$, and $\nu$ are determined exactly,

\section{B. The truncated 4-simplex lattice}

The analysis for this lattice is very similar to that of the truncated 3-simplex lattice discussion in $\mathrm{A}$. We define the restricted partition functions $A^{(r)}, B^{(r)}$, $C^{(r)}, D^{(r)}, E^{(r)}$, and $F^{(r)}$, for the $r$ th order square, as shown in Fig. 4. Due to the permutation symmetry between the vertices of the rth order square, only six different restricted portion functions are needed. The starting values of these weights are

$$
\begin{aligned}
& A^{(0)}=x, \\
& C^{(0)}=\sqrt{x}, \\
& B^{(0)}=D^{(0)}=E^{(0)}=F^{(0)}=0 .
\end{aligned}
$$

The recursion equations for these weights are written down by constructing graphically all possible ways an $(r+1)$ th order square may be constructed out of its constituting $r$ th order squares. We get

$$
\begin{aligned}
A^{(r+1)}= & A^{2}+2 A^{3}+2 A^{4}+4 A^{3} B+6 A^{2} B^{2} \\
B^{(r+1)}= & A^{4}+4 A^{3} B+22 B^{4}, \\
C^{(r+1)}= & C\left(1+3 A+6 A^{2}+6 A^{3}+6 A^{2} B\right)+D\left(6 A^{2}+12 A^{3}\right. \\
& \left.+18 A^{2} B\right) \\
D^{(r+1)}= & C\left(A^{2}+2 A^{3}+3 A^{2} B\right)+D\left(3 A^{2}+7 A^{3}+16 A^{2} B\right. \\
& \left.+22 B^{2} A+22 B^{3}\right),
\end{aligned}
$$

where again the superscripts $(r)$ on each term in the right-hand side of Eq. (38) have been suppressed. We have not written down the explicit expressions for $E^{(r+1)}$ and $F^{(r+1)}$ because, as with $\mathrm{Eq}$. (16d), they are not needed for the determination of critical exponents. The expressions for $E^{(r+1)}$ and $F^{(r+1)}$ involve a sum of terms that are linear in $E^{(r)}$ and $F^{(r)}$ but independent of $C^{(r)}$ and $D^{(r)}$, and terms that are quadratic in $C^{(r)}$ and $D^{(r)}$ but independent of $E^{(r)}$ and $F^{(r)}$. The asymptotic behavior of $E^{(r)}$ and $F^{(r)}$ is determined by the rate at which $C^{(r)}$ and $D^{(r)}$ grow with $r$ 。

Explicit expression for $P(x)$ is easily written down, 


$$
P(x)=\sum_{r=1}^{\infty} 4^{-r}\left\{4 A^{(r-1)^{3}}+3 A^{(r-1)^{4}}\right\},
$$

and a similar but more complicated expression for $C(x)$. Again we notice that the recursion equations are quite simple. $A^{(r+1)}$ and $B^{(r+1)}$ depend only on $A^{(r)}$ and $B^{(r)}$. For any given value of $x$, we can determine $P(x)$ with the help of Eqs. (37)-(39). If $x$ is less than $1 / \mu$, where $\mu$ is now the connective constant for this lattice, $A^{(r)}$ and $B^{(r)}$ tend to zero for large $r$, and $P(x)$ is

finite. The reverse is the case if $x>\mu^{-1}$. This allows a very easy determination of $\mu$ numerically. We start with the obvious bounds $x_{\min }<1 / \mu<x_{\max }$ with $x_{\min }$ $=\frac{1}{3}$ and $x_{\max }=1$. Determination of the behavior of $P(x)$ for $x=\left(x_{\min }+x_{\max }\right) / 2$ allows us to reduce the range of uncertainty $\left(x_{\max }-x_{\min }\right)$ by a factor of 2 . The procedure may be repeated till any arbitrary desired accuracy is obtained. Numerically, we find that

$$
\mu \cong 2.2866 \text {. }
$$

Equations (38a) and (38b) have a fixed point given by

$$
A^{*} \cong 0.4294 \text { and } B^{*} \cong 0.04998 \text { 。 }
$$

There are other fixed points of these recursion equations. The fixed points $A^{*}=B^{*}=0$ and $A^{*}=B^{*}=\infty$ are the trivial attractive fixed points. Other fixed points of Eqs. (38a) and (38b) are not relevant for the determination of the critical behavior because if we start with $x=1 / \mu$, the successive values of $A^{(r)}$ and $B^{(r)}$ tend to the fixed point given by Eq. (41) and hence this is the fixed point which determines the critical behavior of the generating functions $C(x), P(x)$, and $R(x)$.

Let us write

$$
\begin{aligned}
& A^{(r)}=A^{*}+\delta A^{(r)}, \\
& B^{(r)}=B^{*}+\delta B^{(r)} .
\end{aligned}
$$

Then to first order in $\delta A$ and $\delta B$, we have the linearized recursion equations

$$
\begin{aligned}
& \delta A^{(r+1)}=T_{11} \delta A^{(r)}+T_{12} \delta B^{(r)}, \\
& \delta B^{(r+1)}=T_{21} \delta A^{(r)}+T_{22} \delta B^{(r)} .
\end{aligned}
$$

Here $T_{i j}$ are the elements of a $(2 \times 2)$ matrix whose value in terms of $A^{*}$ and $B^{*}$ is easily written down, and its eigenvalues and eigenvectors determined. We find that the matrix $T$ has the eigenvalues

$$
\begin{aligned}
& \lambda_{1} \cong 2.7965, \\
& \lambda_{2} \cong 0.2538 .
\end{aligned}
$$

We note that only one of the eigenvalues is greater than zero. This implies that

$$
\begin{aligned}
& \delta A^{(r)} \cong K a_{1} \lambda_{1}^{r} \delta, \\
& \delta B^{(r)} \cong K a_{2} \lambda_{1}^{r} \delta,
\end{aligned}
$$

where $\left(a_{1}, a_{2}\right)$ is the eigenvector corresponding to the eigenvalue $\lambda_{1}$. $\delta$ is defined equal to $\left(\mu^{-1}-x\right)$ and $K$ is some constant of proportionality same for EqS. (45a) and (45b). These equations hold when $r$ is not too large (so that the linear approximation is adequate) and not too small (so that the "irrelevant" part of $\delta A$ and $\delta B$, which is proportional to $\lambda_{2}$ is negligible). Let us denote by $P^{\text {sing }}(1 / \mu-\delta)$ the singular part of $P(1 / \mu-\delta)$. Then from Eq. (39) we get

$$
P^{\operatorname{sing}}\left(\frac{1}{\mu}-\delta\right) \approx \frac{1}{4} P^{\sin 8}\left(\frac{1}{\mu}-\lambda_{1} \delta\right) .
$$

Assuming that

$$
P^{\operatorname{sing}}\left(\frac{1}{\mu}-\delta\right) \sim \delta^{2-\alpha}
$$

we get

$$
\alpha=2-\ln 4 / \ln \lambda_{1} \simeq 0.5413 .
$$

Again we choose a small positive number $\epsilon$, and choose a value of $x$ sufficiently close to $\mu^{-1}$ so that

$$
1 \gg \epsilon \gg \delta
$$

and

$$
r_{0} \stackrel{\text { def }}{=} \ln (\epsilon / \delta) / \ln \lambda_{1} .
$$

Then for $r<r_{0}$ we have $A^{(r)} \approx A^{*}, B^{(r)} \approx B^{*}$ and the recursion equations for $C^{(r)}$ and $D^{(r)}$ become, from Eqs. (38c) and (38d),

$$
\begin{aligned}
C^{(r+1)} \approx & C^{(r)}\left(1+3 A^{*}+6 A^{*^{2}}+6 A^{*^{3}}+6 A^{*^{2}} B^{*}\right. \\
& +D^{(r)}\left(6 A^{*^{2}}+12 A^{*^{3}}+18 A^{*^{2}} B^{*}\right), \\
D^{(r+1)} \approx & C^{(r)}\left(A^{*^{2}}+2 A^{*^{3}}+3 A^{*^{2}} B^{*}\right) \\
& +D^{(r)}\left(3 A^{*^{2}}+7 A^{*^{3}}+16 A^{*^{2}} B^{*}+22 B^{*^{2}} A^{*}+22 B^{*^{3}}\right) .
\end{aligned}
$$

These are linear recursion equations and show that, for $r<r_{0}, C^{(r)}$ and $D^{(r)}$ increase as $\lambda_{+}^{r}$, where $\lambda_{+}$is the larger eigenvalue of the matrix that characterizes the linear transformation of $\mathbf{E q}$. (50). Numerically, substituting the values of $A^{*}$ and $B^{*}$ we find that

$$
\lambda_{+} \cong 4.2069 \text {. }
$$

For $r>r_{0}$, the coefficients $A^{(r)}, B^{(r)}$, and $D^{(r)}$ rapidly approach zero and $C^{(r)}$ tends to its asymptotic value which is proportional to $\lambda_{+}^{\gamma_{0}}$. The argument as before shows that

$$
C(x) \sim K\left(\lambda_{+}^{2} / 4\right)^{r_{0}} \text { 。 }
$$

Substituting for $r_{0}$ from Eq。(49) and comparing with $\mathrm{Eq}$. (7) we get

$$
\gamma=\ln \left(\lambda^{2} / 4\right) / \ln \left(\lambda_{1}\right) \approx 1 。 4461 .
$$

And since

$$
R(x) \sim K\left(2^{r_{0}}\right)^{2}\left(\lambda_{+}^{2} / 4\right)^{r_{0}}
$$

we get from Eqs. (49) and (9)

$$
\nu=\ln 2 / \ln \lambda_{1} \approx 0.7294 \text {. }
$$

\section{The modified rectangular lattice}

The restricted partition functions for the $r$ th order block ${ }^{13}$ of lattice sites are defined in Fig。 5. We have shown only the configuration with no or only one end point of the walk. As before, the restricted partition functions with two end points of restricted walks, may be defined and are necessary to calculate the generating 

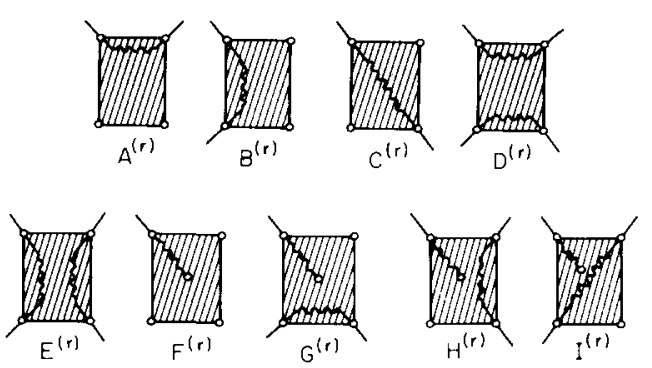

FIG. 5. Restricted partition functions for the $r$ th order block of lattice sites of a modified rectangular lattice. Only the restricted partition functions correspondings to zero or one end points of the self avoiding walk are shown.

function $C(x)$, but do not affect the critical exponents. The recursion equations are complicated by the fact that the number of coupling constants is much larger than in the previous two cases. This is, of course, more representative of real life renormalization calculations where the total number of coupling constants is in principle infinite.

The starting values of the different weights are

$$
\begin{aligned}
& A^{(1)}=B^{(1)}=x^{2}+x^{4}, \\
& C^{(1)}=2 x^{3}, \\
& D^{(1)}=E^{(1)}=x^{4}, \\
& F^{(1)}=x^{1 / 2}\left[1+2 x+2 x^{2}+2 x^{3}\right], \\
& G^{(1)}=H^{(1)}=x^{5 / 2}(1+x), \\
& I^{(1)}=x^{7 / 2} .
\end{aligned}
$$

Again we may write down the recursion equations for these weights by drawing all possible configurations of self avoiding random walks on the $(r+1)$ th order block. Figure 6 shows the configurations that contribute to $C^{(r+1)}$. Other recursion equations are similarly written down. We get

$$
\begin{aligned}
& A^{(r+1)}=B(1+D), \\
& B^{(r+1)}=A^{2}+C^{2}, \\
& C^{(r+1)}=2 A C, \\
& D^{(r+1)}=B^{2}+2 D E, \\
& E^{(r+1)}=D^{2} \\
& F^{(r+1)}=F(1+A+C)+G B+I B, \\
& G^{(r+1)}=F B+G E+I E+H(D+A+C), \\
& H^{(r+1)}=G(A+D)+I C, \\
& I^{(r+1)}=G C+I(A+D),
\end{aligned}
$$

where again we have suppressed the superscripts $(r)$ in the right-hand sides of $\mathrm{Eq}$. (57). We have quite a simple expression of $P(x)$,
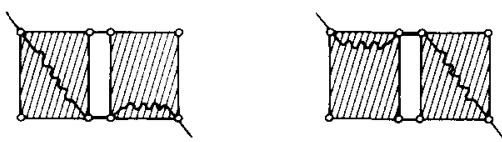

FIG. 6. Configurations of random walks that contribute to $C^{(r+1)}$ for the modified rectangular lattice. The shaded rectangles are the $r$ th order blocks with only the corner vertices and the walk going through them shown.

$$
P(x)=\sum_{r=1}^{\infty}\left(B^{(r)}\right)^{2} / 2^{r+2}+\frac{x^{4}}{4} .
$$

Numerically, we find the largest value of $x$ for $P(x)$ to converge is

$$
x_{c} \cong 0.5914
$$

which corresponds to the connective constant

$$
\mu=1 / x_{c} \approx 1.6909 \text {. }
$$

If we start with the value $x=\mu^{-1}$, the sequence $\left(A^{(r)}\right.$, $\left.B^{(r)}, C^{(r)}, D^{(r)}, E^{(r)}\right)$ converge to the fixed point

$$
\text { (0.5000, 0.4201, 0.4124,0.1902, 0.0362). }
$$

Linearizing the recursion equations about this fixed point, we find the eigenvalues of the transformation matrix. Only one eigenvalue is larger than 1. Numerically, its value is

$$
\lambda_{1} \cong 1.6839 \text {. }
$$

Thus arguing as before, we find

$$
\alpha=2-\frac{\ln 2}{\ln \lambda_{1}} \approx 0.6699 \text {. }
$$

Substituting for $A^{(r)}, B^{(r)}, C^{(r)}, D^{(r)}$, and $E^{(r)}$ the fixed point values given by $(61)$ in the recursion equations (57f)-(57i), we find numerically, the largest eigenvalue of the corresponding transformation matrix. This is found to be

$$
\lambda_{+} \approx 2.0582 \text {. }
$$

Then analogous to $\mathrm{Eq}$. (53) we find

$$
\gamma=\ln \left(\lambda_{+}^{2} / 2\right) / \ln \lambda_{1} \approx 1.4403 \text { 。 }
$$

The diameter of an $(r+2)$ th order block is twice the diameter of an $r$ th order block, the diameter being defined as the largest distance between any two points in the block. Thus, arguing as for the truncated 4simplex lattice, we get

$$
\nu=(\ln 2) / 2 \ln \lambda_{1} \approx 0,6650
$$

which determines all the critical exponents for the lattice.

\section{DISCUSSION}

We note that in all the cases considered, the critical indices satisfy the relation

$$
d \nu=2-\alpha,
$$

where $d$ is the dimensionality of the lattice as defined by Nelson and Fisher. On the other hand, we know that in other cases (e.g., the $X Y$ model discussed in I) the dimensionality of the lattice is more usefully defined by the power law behavior of the cumulative frequency 


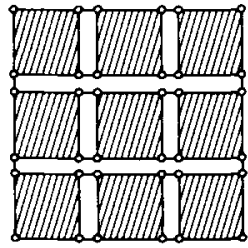

FIG. 7. A generalization of the modified rectangular lattice. The figure shows the graph of an $(r+1)$ th order rectangle. Shaded rectangles denote $r$ th order graphs of which only the corner vertices are shown. The case depicted is $p=3$.

distribution function for low frequencies. Perhaps the self avoiding random walks are atypical in that the generating functions whose singularities determine the critical exponents are not given in terms of the partition function of a Hamiltonian. The critical behavior of the self avoiding random walks depends strongly on the connectivity properties of the lattice and not on the dimensionality alone. For example, we expect the critical indices $\alpha, \gamma, \nu$ to be different for the self avoiding walks for planar and nonplanar two-dimensional lattices. This is because planarity determines if the walk can cross itself or not. On the other hand, the critical exponents for the Ising model in two dimensions are expected to remain unchanged if a small nextnearest neighbor interaction is added to the original nearest neighbor Hamiltonian (which makes the lattice nonplanar).

It is possible to construct pseudolattices that have the same effective dimensionality, but different critical exponents for the self avoiding walk problem. Consider, for example, the lattice defined in Fig. 7. This is a simple generalization of the modified rectangular lattice. The first order rectangle is a cyclic graph on four points. An $(r+1)$ th order rectangle is formed by taking $p^{2} r$ th order rectangles ( $p$ is any integer $>1$ ) and arranging them in a $p \times p$ array. We connect the rectangles in the same row by horizontal bonds connecting the corner vertices of adjacent rectangles in the same adjacent rectangles in the same row. Finally $2(p-1)$ vertical bonds are added to connect the corner vertices of adjacent rows. In Fig. 7 the construction is illustrated for the case $p=3$.

It is easy to see that the lattice is planar and has coordination number 3 . Using the same method as used to determine the effective dimensionality of the modified rectangular lattice in $I$, it may be shown that the effective dimensionality of the lattice is $\frac{3}{2}$, independent of $p$. The modified rectangular lattice corresponds to the special case $p=2$. Also, we see that the dimensionality of the lattice is 2 (again independent of $p$ ) if we use Nelson and Fisher's definition. Though the coordination number and the dimensionality of the lattice is independent of $p$ (whichever definition of dimensionality is used), it is easy to verify that the critical exponents for the self avoiding walks on these lattices do depend on $p$. In particular for $p=3$ we find

$$
\alpha=0.6589, \quad \gamma=1.4601, \nu=0.6705
$$

which differs from the exponents for $p=2$ (Eq. (12b).

It appears that the connectivity structure of these lattices is quite complicated, and a single value of "effective dimensionality" is not sufficient to completely characterize the critical behavior of self avoiding walks on such lattices. More study in this area is needed to identify the parameters that can be used to completely characterize the critical behavior of different systems on such pseudolattices.

\section{ACKNOWLEDGMENTS}

It is a pleasure to thank Professor Jon Mathews for many discusssions and critical comments, and Ms. RLou Norquist for typing the manuscript.

'E.W. Montroll, J. Chem. Phys, 18, 734 (1950).

${ }^{2}$ M. E. Fisher and M. F. Sykes, Phys. Rev. 114, 45 (1959).

${ }^{3}$ D.S. McKenzie, Phys. Rep. C 27, 37 (1976); and references cited therein.

${ }^{4}$ S. G. Whittington and J. F. Harris, J. Phys. A 5, 411 (1972); C. Domb and F. T. Hioe, J. Chem. Phys. 51, 1920 (1969).

${ }^{5}$ M. F. Sykes et al. , J. Phys. A: Gen. Phys. 5, 653 (1972); F. T. Wall and F. Mandel, J. Chem. Phys. 63, 4592 (1975). ${ }^{6} \mathrm{E}$. Muller-Hartmann and J. Zittartz, Phys. Rev. Lett. 33, 893 (1976).

7J. Zittartz, Z. Phys. Chem. Chem. Abt. B 23, 55-63 (1976).

${ }^{8} \mathrm{~K}$. Wilson and J. Kogut, Phys. Rep. C 12, 75 (1974).

${ }^{9}$ This is discussed in Ref. 8.

${ }^{10} \mathrm{G}$. Gallavotti and H. Knopps, Nuovo Cimento 5, 341 (1975).

${ }^{11}$ D. R. Nelson and M.E. Fisher, Ann. Phys. (N. Y.) 91, 226 (1975).

${ }^{12}$ D. Dhar, J. Math. Phys. 18, 577 (1977).

${ }^{13}$ See page 581 of Ref. 12 for the definition of a block of sites. 